International Journal of Advanced Astronomy, 7 (2) (2019) 49-56
International Journal of Advanced Astronomy
SPC
Website: $w w w . s c i e n c e p u b c o . c o m / i n d e x . p h p / I J A A$
Research paper

\title{
Heterogeneous primaries in CR4BP
}

\author{
Abdullah A. Ansari ${ }^{1 *}$, Ashraf Ali ${ }^{2,1}$, Kumari Shalini ${ }^{3,1}$, Mehtab Alam ${ }^{1}$ \\ ${ }^{1}$ International Center for Advanced Interdisciplinary Research (ICAIR), Ratiya Marg, Sangam Vihar, New Delhi, India \\ ${ }^{2}$ Department of Computer Science and Information, College of Science Al-Zulfi, Majmaah University, KSA \\ ${ }^{3}$ Department of Mathematics, Zakir Hussain Delhi College, University of Delhi, India \\ *Corresponding author E-mail: abdullah2008@ rediffmail.com
}

\begin{abstract}
This paper investigates the motion of the massless body moving under the influence of the gravitational forces of the three equal heterogeneous oblate spheroids placed at Lagrangian configuration. After determining the equations of motion and the Jacobian constant of the massless body, we have illustrated the numerical work (Stationary points, zero-velocity curves, regions of motion, Poincare surfaces of section and basins of attraction). And then we have checked the linear stability of the stationary points and found that all the stationary points are unstable.
\end{abstract}

Keywords: Equilateral Triangle; Equilibrium Points; Heterogeneous Primaries; R4BP; Stability.

\section{Introduction}

The restricted four-body problem (R4BP) is an extension of the restricted three-body problem (R3BP) in the field of Celestial Mechanics in which three bodies with different masses, as called primaries, orbits (circular or elliptic) around their center of mass due to their Newtonian gravitational forces, and the fourth body with negligible mass is moving in the plane of the primaries which is attracted by the primaries and influenced by their motion but not influencing them. In the extended R4BP there exist ten libration points out of which four are collinear and rests are non-collinear. The collinear libration points are unstable while non-collinear libration points are stable with some restrictions.

In recent times, the R4BP has been studied by various researchers with many perturbations. Kalvouridis et al. (2006 a, b) have determined the dynamical model of the R4BP under radiation pressure. Papadakis (2007, 2016 a, b) has investigated the asymptotic orbits in the R4BP. Baltagiannis et al. (2011 a, b) have studied the equilibrium points and their stability in the R4BP and they further discussed on the periodic orbits in the same configuration. Ceccaroni (2012) has studied on low-thrust propulsion in a coplanar circular R4BP. Papadouris et al. $(2013,2014)$ have determined equilibrium points in the photo-gravitational R4BP. Kumari et al. (2013, 2014) have investigated the equilibrium points and zero velocity surfaces in the R4BP under the effect of solar wind drag and they further studied the stability regions of equilibrium points under the effect of oblateness. Equilibrium, in and out-of-plane in the symmetric collinear R4BP under radiation pressure have been studied by Arribas, et al. (2016 a, b). Asique et al. (2015 a, b, 2016, 2017) have discussed on the R4BP by taking the third primary as an oblate spheroid, tri-axial rigid body and ellipsoid and they further studied the same problem under the effect of photo-gravitational. Singh et al. (2015 a, b) have determined the out-of-plane equilibrium points in the photo-gravitational R4BP and they further studied the effect of perturbations in the Coriolis and centrifugal forces on the stability of equilibrium points. Singh et al. (2016 a, b) have studied the equilibrium points in the R4BP with radiation pressure. Suraj et al. (2017 a, b) have investigated the R4BP under the combine effect of small perturbations in the Coriolis and centrifugal forces with different shapes of the primaries. Zotos (2017) studied the basins of convergence in the equilateral R4BP.

Esteban and Vazquez (2001) were the first who introduced the Heterogeneous body, they have taken a stratified non-conformal heterogeneous oblate spheroidal system with three layers. Suraj et al. (2014) have determined the photo-gravitational R3BP when the primaries are heterogeneous spheroid with three layers. Idrisi et al. (2016) have studied the non-collinear libration points in circular R3BP when the less massive primary is a heterogeneous oblate body with N-layers. Shalini et al. (2017) have discussed the non-linear stability of L4 in the R3BP when the smaller primary is a heterogeneous spheroid with three layers having different densities. It is observed that in the nonlinear sense, the triangular libration is stable in the range of linear stability $0<\mu<\mu_{\mathrm{c}}$, a critical value of mass parameter $\mu$, except for three mass ratios $\mu_{1}, \mu_{2}, \mu_{3}$ at which Moser's theorem is not applicable. Ansari et al. (2014, 2016 a, b, c, 2017, 2018) have numerically illustrated the equilibrium points, zero-velocity curves, regions of motion and basins of attraction in the R3BP and R4BP by considering the different shapes of the primaries.

This study is organized as follows: In the second section mathematical model with the statement, the equations of motion and Jacobi integral have studied. In the third section numerical performances, we have done the graphical work with the software Mathematica. In the next and fourth section, the linear stability of the equilibrium points has been examined and finally the paper ends with conclusion. 


\section{Mathematical model with statement}

Let three heterogeneous oblate primaries $\mathrm{m}_{1}, \mathrm{~m}_{2}$ and $\mathrm{m}_{3}$ of equal masses be placed at the vertices of an equilateral triangle $\mathrm{ABC}$ with coordinates as

$\left(x_{1}, 0,0\right)=\left(\frac{\ell}{\sqrt{3}}, 0,0\right),\left(x_{2}, y_{2}, 0\right)=\left(-\frac{\ell}{2 \sqrt{3}}, \frac{\ell}{2}, 0\right), \quad\left(x_{3}, y_{3}, 0\right)=\left(-\frac{\ell}{2 \sqrt{3}},-\frac{\ell}{2}, 0\right)$.

These primaries are moving in circular orbits around their common center of mass $\mathrm{O}$ which is taken as the origin. The fourth mass $\mathrm{m}$ which is much less than the masses of the primaries is moving in the space under the gravitational forces $\vec{F}_{1}, \vec{F}_{2}$ and $\vec{F}_{3}$ of the heterogeneous oblate primaries but not influencing them.

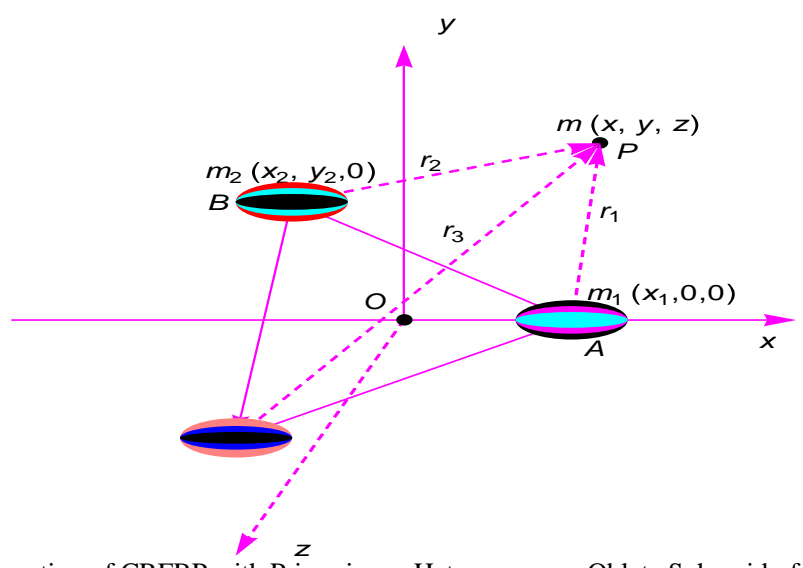

Fig. 1: Configuration of CRFBP with Primaries as Heterogeneous Oblate Spheroid of Three Layers.

Let $\vec{r}_{1}, \vec{r}_{2}$ and $\vec{r}_{3}$ be the distances of the fourth body from the primaries. This is also considered that the principal axes of the heterogeneous oblate body remain parallel to the synodic axes Oxyz throughout the motion and the synodic coordinate system coincides initially with the inertial coordinate system, and revolving with angular velocity $\omega=\mathrm{n} k$, about the $\mathrm{z}$-axis. Where $\mathrm{n}$ is the magnitude of $\omega$ (Fig. 1). The equation of motion of $m$ in the vector form can be written as:

$m\left(\frac{\partial^{2} \vec{r}}{\partial t^{2}}+2 \vec{\omega} \times \frac{\partial \vec{r}}{\partial t}+\frac{\partial \vec{\omega}}{\partial t} \times \vec{r}+\vec{\omega} \times(\vec{\omega} \times \vec{r})\right)=\vec{F}$.

Where, $\vec{r}=\overrightarrow{O P}, \vec{F}=$ the total force acting on the infinitesimal body due to the primaries,

$\frac{\partial^{2} \vec{r}}{\partial t^{2}}=\ddot{x} i+\ddot{y} j+\ddot{z} k=$ Relative acceleration,

$2 \vec{\omega} \times \frac{\partial \vec{r}}{\partial t}=-2 n \ddot{y i}+2 n \ddot{x j}=$ Coriolis acceleration,

$\frac{\partial \vec{\omega}}{\partial t} \times \vec{r}=0=$ Euler's acceleration,

$\vec{\omega} \times(\vec{\omega} \times \vec{r})=-n^{2} x i-n^{2} y j=$ Centrifugal acceleration

The gravitational potential of the heterogeneous oblate spheroid with three layers of densities $\rho_{p}$ and axes

$\left(a_{p}, b_{p}, c_{p}\right), \rho_{p}<\rho_{p+1}, a_{p}<a_{p+1}, b_{p}<b_{p+1}, c_{p}<c_{p+1}$ at any point is

$V=-\frac{\text { mass } G}{R}-\frac{f G}{2 R^{3}}+\frac{3 f G z^{2}}{2 R^{5}}$,

Where, $\mathrm{R}=$ Distance between heterogeneous oblate body and the point at which potential is going to be found.

$f=\frac{4 \pi}{3} \sum_{p=1}^{3}\left(\left(\rho_{p}-\rho_{p+1}\right) a_{p} b_{p} c_{p} \sigma_{p}\right)$

$\sigma_{p}=\frac{\left(a_{p}^{2}-c_{p}^{2}\right)}{5}, \rho_{4}=0 . \quad$ Ansari 2018) 
Hence, the whole potential at point $\mathrm{P}$ due to $m_{1}, m_{2}$ and $m_{3}$ is

$V=\sum_{i=1}^{3}\left(-\frac{m_{i} G}{r_{i}}-\frac{f_{i} G}{2 r_{i}^{3}}+\frac{3 f_{i} G z^{2}}{2 r_{i}^{5}}\right)$.

Let us fix the units such that the sum of the masses $\mathrm{m}_{1}+\mathrm{m}_{2}+\mathrm{m}_{3}=1 \Rightarrow m_{1}=\frac{1}{3}$ (because all masses are equal), the distance between two heterogeneous oblate primaries be equal to unity i.e. $\ell=1$ and unit of time is so considered that $\mathrm{G}=1$ and $\mathrm{n}=1$.

Then from equation (1), the equations of motion of the fourth body in dimensionless variables can be rewritten as

$\ddot{x}-2 \dot{y}=\frac{\partial \prod}{\partial x}$,

$\ddot{y}+2 \dot{x}=\frac{\partial \prod}{\partial y}$,

$\ddot{z}=\frac{\partial \prod}{\partial z}$,

Where,

$\Pi=\frac{1}{2}\left(x^{2}+y^{2}\right)+\sum_{i=1}^{3}\left(\frac{1}{3 r_{i}}+\frac{f_{i}}{2 r_{i}^{3}}+\frac{3 f_{i} z^{2}}{2 r_{i}^{5}}\right)$,

$r_{i}^{2}=\left(x-x_{i}\right)^{2}+\left(y-y_{i}\right)^{2}+z^{2}$,

$f_{i}=\frac{4 \pi}{3} \sum_{p=1}^{3}\left(\left(\rho_{p}^{i}-\rho_{p+1}^{i}\right) a_{p}^{i} a_{p}^{i} c_{p}^{i} \sigma_{p}^{i}\right)$

$\sigma_{p}^{i}=\frac{\left(a_{p}^{i 2}-c_{p}^{i 2}\right)}{5}, \rho_{4}^{i}=0 .(i=1,2,3)$

And hence, from equation (3), the Jacobi-Integral can be written as

$\dot{x}^{2}+\dot{y}^{2}+\dot{z}^{2}=2 \prod-C$.

Where $\mathrm{C}$ is the Jacobi Integral constant that is conserved and associated with the whole energy of the system. We have represented the curve for a particular value of the energy integral which is restricted with its motion to the regions where $C \geq 2 \Pi$, and all other areas are forbidden to the fourth body.

\section{Numerical performance}

In this section, we have located the stationary points in three planes (x-y, y-z, x-z-planes), and plotted the zero velocity curves, regions of possible motion, Poincare surfaces of section and basins of attraction under the effect of three heterogeneous oblate primaries. (In complete calculations, we have taken $\mathrm{f}_{1}=9.83933 \times 10^{-18}, \mathrm{f}_{2}=1.58302 \times 10^{-7}, \mathrm{f}_{3}=3.13153 \times 10^{-8}$. (Ansari 2018))

\subsection{Positions of stationary points}

The positions of stationary points can be found by solving the right side of equations (3) when all the derivatives are zero.i.e.

$\frac{\partial \Pi}{\partial x}=0, \quad \frac{\partial \Pi}{\partial y}=0, \frac{\partial \Pi}{\partial z}=0$.

Here, we have illustrated the locations of stationary points in (x-y)-plane (fig 2a), (y-z)-plane (fig2b) and (x-z)-plane (fig2c). And in (x-y)-plane found 10 stationary points in which four are collinear $\left(\mathrm{L}_{1}, \mathrm{~L}_{2}, \mathrm{~L}_{3}\right.$ and $\left.\mathrm{L}_{4}\right)$ and rest six are non-collinear $\left(\mathrm{L}_{5}, \mathrm{~L}_{6}, \mathrm{~L}_{7}, \mathrm{~L}_{8}, \mathrm{~L}_{9}\right.$, and $\left.\mathrm{L}_{10}\right)$ We observed that $\mathrm{L}_{1}$ and $\mathrm{L}_{2}$ are symmetrical about the $\mathrm{y}$-axis and $\mathrm{L}_{5}, \mathrm{~L}_{7}$ and $\mathrm{L}_{9}$ are symmetrical about the $\mathrm{x}_{-}$axis to $\mathrm{L}_{6}$, $\mathrm{L}_{8}$, and $\mathrm{L}_{10}$ respectively. Red color points are positions of the primaries $\left(m_{1}, m_{2}, m_{3}\right)$ which are located at the vertices of an equilateral triangle. The complete view of the stationary points is looked like the cardioid shape.

In (y-z)- plane, we found five collinear stationary points $\left(\mathrm{L}_{1}, \mathrm{~L}_{2}, \mathrm{~L}_{3}, \mathrm{~L}_{4}\right.$, and $\left.\mathrm{L}_{5}\right)$. Where $\mathrm{L}_{3}$ is located at the origin while $\mathrm{L}_{1}$, $\mathrm{L}_{2}$ are located at left side of the origin and $\mathrm{L}_{4}, \mathrm{~L}_{5}$ are located at right side of the origin. And also we observed that $\mathrm{L}_{1}, \mathrm{~L}_{2}$ are symmetrical about the $\mathrm{z}$-axis to $\mathrm{L}_{4}, \mathrm{~L}_{5}$ respectively. The complete view of the stationary points is looked like kidney shape.

Similarly, in $\mathrm{x}-\mathrm{z}$-plane, we found four collinear stationary points $\left(\mathrm{L}_{1}, \mathrm{~L}_{2}, \mathrm{~L}_{3}\right.$ and $\left.\mathrm{L}_{4}\right)$ on the $\mathrm{x}$-axis. Where $\mathrm{L}_{3}$ is located at origin while $\mathrm{L}_{1}$, $\mathrm{L}_{2}$, and $\mathrm{L}_{4}$ are located left and right side of the origin. The complete view of the stationary points is looked like the different size of kidney shapes. 
(A)

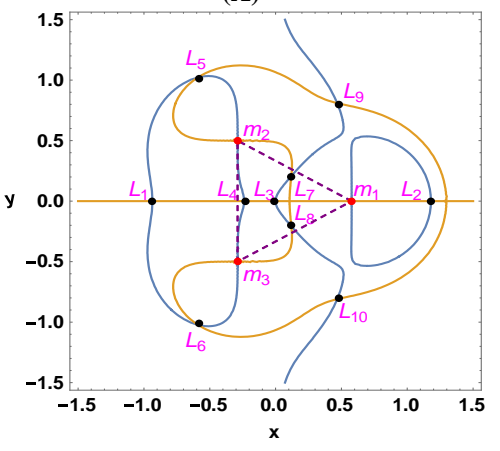

(B)

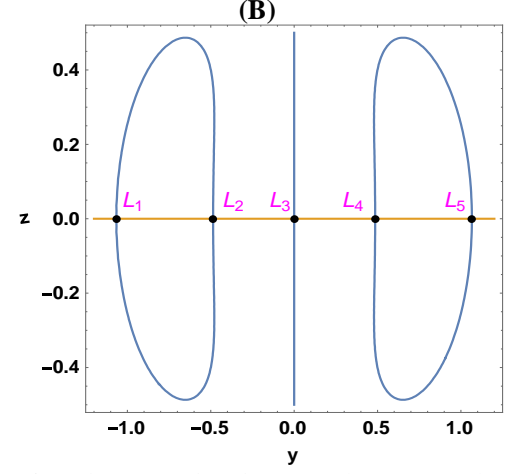

(C)

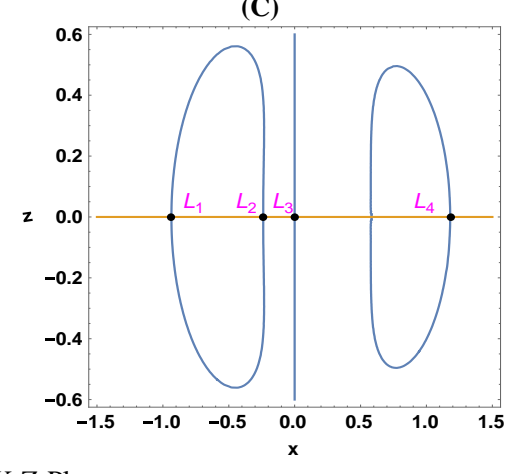

Fig. 2: Locations of Stationary Points in X-Y-Plane, Y-Z-Plane and X-Z-Plane.

\subsection{Zero-velocity curves}

In this subsection, we have illustrated the zero-velocity curves in (x-y)-plane (fig 3a), (y-z)-plane (fig 3b), (x-z)-plane (fig 3c). Firstly, we have evaluated the values of Jacobian constant $(\mathrm{C})$ at all the positions of the stationary points and then we have plotted the zero-velocity curves at all the corresponding Jacobian constants $\left(\mathrm{C}_{\mathrm{L} 1----} \mathrm{C}_{\mathrm{L} 10}\right)$. Each zero-velocity curves corresponding to Jacobian constant are indicated clearly in figures $(3 a, b, c)$ with colors.

(A)

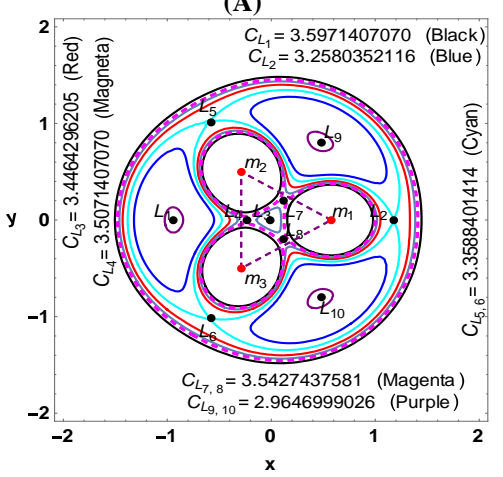

(B)

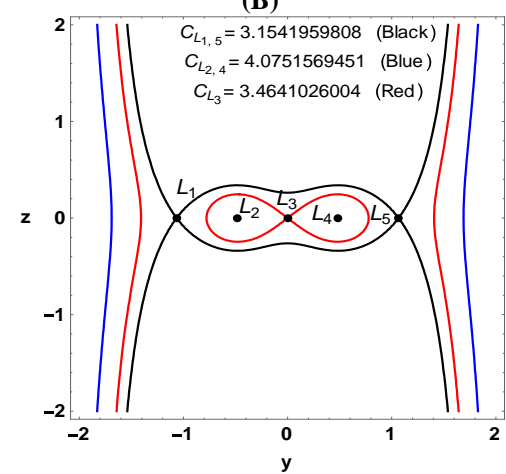

(C)

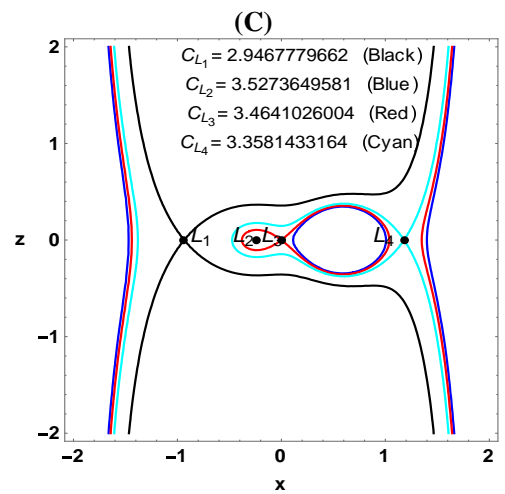

Fig. 3: Zero-Velocity Curves in X-Y-Plane (A), Y-Z-Plane(B) and X-Z-Plane(C).

\subsection{Possible regions of motion}

We have plotted the regions of possible motion with the help of Jacobian constant given in equation (4). The possible motion of the massless body is bounded to the regions in which $\mathrm{C} \geq 2 \pi$ (Blue color regions) and all other regions are prohibited to it. The regions of motion will give us the dynamical behaviour of the infinitesimal body. From fig (4a) at $\mathrm{C}_{\mathrm{L} 1}=3.5971407070$, we observed that the infinitesimal will move in the circular region except for around all three heterogeneous oblate primaries with some radius. From fig (4b) at $\mathrm{C}_{\mathrm{L} 2}$ $=3.2580352116$, we got that the infinitesimal body will move around $\mathrm{L}_{1}, \mathrm{~L}_{9}$ and $\mathrm{L}_{10}$, which are looking like three islands. From fig (4c) at $\mathrm{C}_{\mathrm{L} 3}=3.4464296205$, we observed that the infinitesimal body will move in the circular region except for the Lagrangian configuration region. From fig (4d) at $\mathrm{C}_{\mathrm{L} 4}=3.5071407070$, we observed that the infinitesimal body will move in circular region and also around $\mathrm{L}_{3}$ in triangular shape but it will not move nearby the primaries. From fig (4e) at $\mathrm{C}_{\mathrm{L} 56}=3.3588401414$, we observed that the infinitesimal body will move in the circular regions except around the Lagrangian configuration where $\mathrm{L}_{2}, \mathrm{~L}_{5}$, and $\mathrm{L}_{6}$ will be like a gateway. From fig (4f) at $\mathrm{C}_{\mathrm{L} 78}=3.5427437594$, we observed that the infinitesimal will move in the circular region except around the primaries. From fig(4g) at $\mathrm{C}_{\mathrm{L} 9,10}=2.964699026$, we observed that the infinitesimal body will move only around L9 and L 10 , and the rest part will be prohibited for it These two regions are looked like two islands which are symmetrical about the $\mathrm{x}$-axis.

(A)

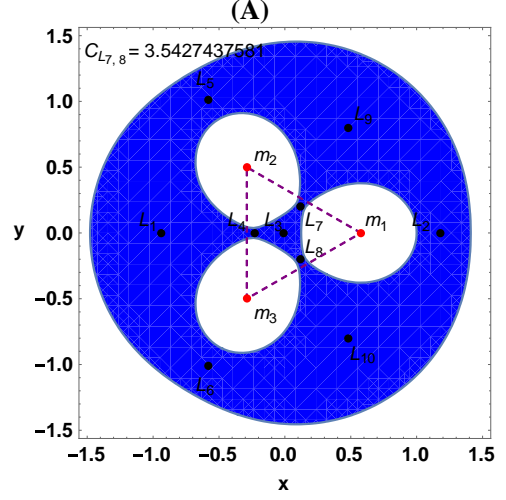

(B)

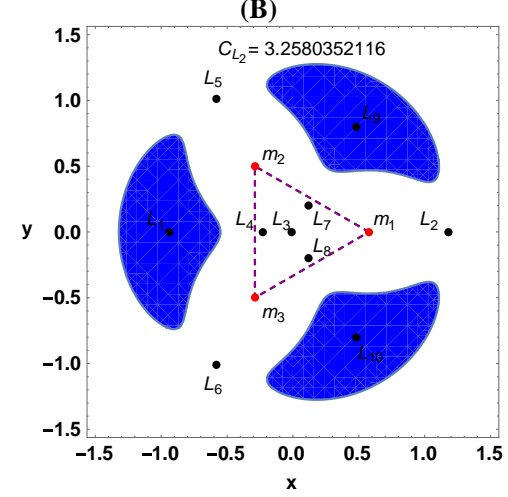

(C)

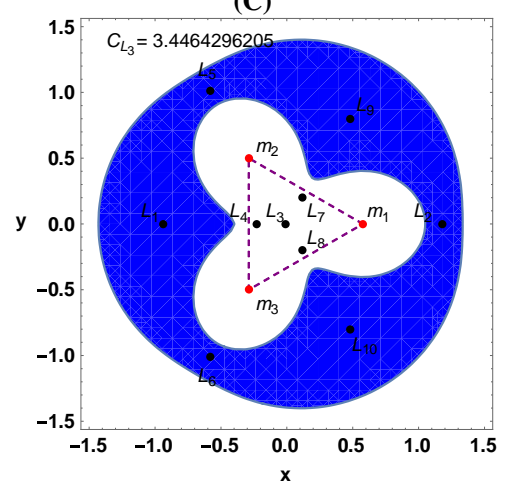


(D)

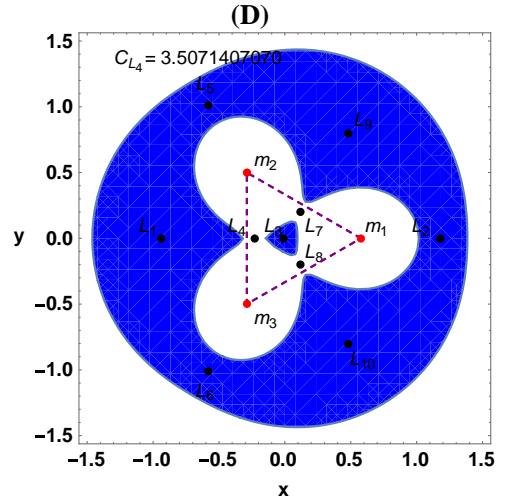

(E)

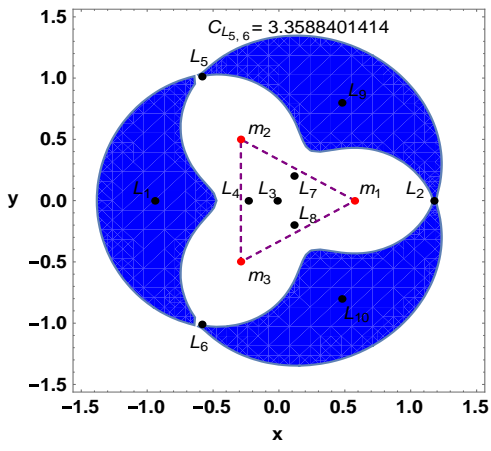

(G)

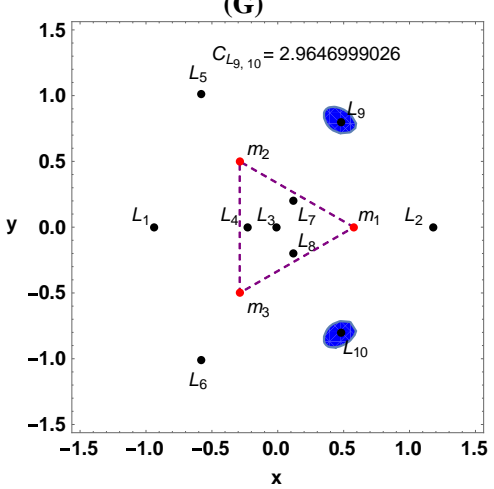

(F)

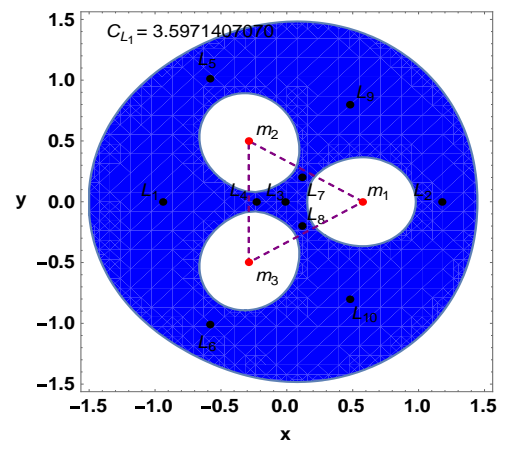

Fig. 4: Regions of Motion in X-Y-Plane Corresponding to Each Stationary Point.

\subsection{Poincare surfaces of section}

We have drawn the Poincare surfaces of the section in $x-x^{\prime}$-plane (fig 5a) and y-y'-plane (fig 5b). From figure 5(a), we observed that the surfaces of section are looking like the shape of a leaf and there is no chaos. While from figure 5(b), we got the shape of the section like lemniscate and also there is no chaos.

(A)

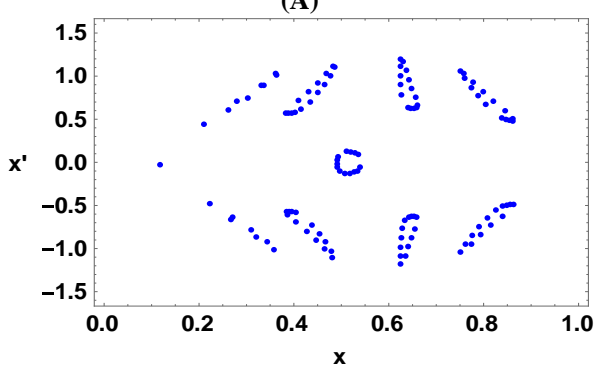

(B)

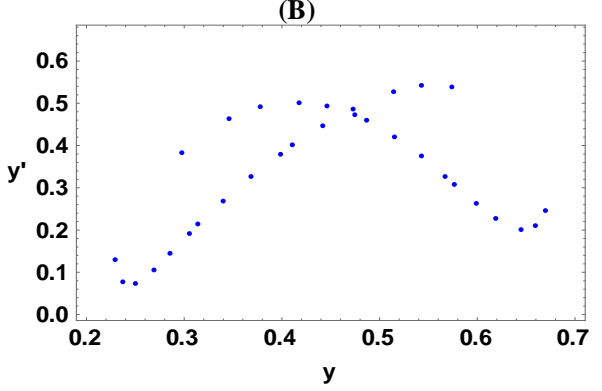

Fig. 5: Poincare Surfaces of Section in $X-X^{\prime}-$ Plane (A) and $Y-Y^{\prime}-P l a n e(B)$.

\subsection{Basins of attraction}

Here we have illustrated the basins of convergence in the frame of the circular R4BP with heterogeneous oblate spheroid of the primaries by using the simple, fast and accurate N-R method. All the specific stationary point are forming the basin of convergence or converging region. To get the basins of attraction is an issue of great importance which shows some most important dynamical behaviour of the system. The formula of our problem in $x-y-$ plane is

$$
\begin{aligned}
& x_{n+1}=x_{n}-\left(\frac{\prod_{x} \prod_{y y}-\prod_{y} \Pi_{x y}}{\prod_{x x} \prod_{y y}-\prod_{x y} \prod_{y x}}\right)_{\left(x_{n}, y_{n}\right)}, \\
& y_{n+1}=y_{n}-\left(\frac{\prod_{y} \prod_{x x}-\prod_{x} \Pi_{y x}}{\prod_{x x} \prod_{y y}-\prod_{x y} \prod_{y x}}\right)_{\left(x_{n}, y_{n}\right)} .
\end{aligned}
$$

Where $\mathrm{x}_{\mathrm{n}}, \mathrm{y}_{\mathrm{n}}$ are the values of $\mathrm{x}$ and $\mathrm{y}$ coordinates of the $\mathrm{n}^{\text {th }}$ step of the N-R iterative process. If the initial point converges rapidly to one of the stationary points then this point $(\mathrm{x}, \mathrm{y})$ will be a member of the basin of convergence of the root. When the successive approximation converges to an attractor, this process stops. For the classification of the stationary points on the planes, we used a color code.

In $x$-y-plane (Fig. 6a), we observed that the stationary points $\mathrm{L}_{1}, \mathrm{~L}_{2}, \mathrm{~L}_{3}$, and $\mathrm{L}_{4}$ represent cyan color regions, $\mathrm{L}_{5}$ and $\mathrm{L}_{6}$ represent red color regions, $\mathrm{L}_{7}$ and $\mathrm{L}_{8}$ represent sky blue and green color regions respectively, and also $\mathrm{L}_{9}$ and $\mathrm{L}_{10}$ represent magenta and orange color regions. The basins of attraction corresponding to the stationary points $\mathrm{L}_{1}, \mathrm{~L}_{2}, \mathrm{~L}_{3}, \mathrm{~L}_{4}, \mathrm{~L}_{5}, \mathrm{~L}_{6}, \mathrm{~L}_{9}$, and $\mathrm{L}_{10}$ extend to infinity while corresponding to $\mathrm{L}_{7}$ and $\mathrm{L}_{8}$ cover finite regions. 
Figure $6 \mathrm{~b}$ represents the zoomed image of the figure $6 \mathrm{a}$ near the Lagrangian configuration.

(A)

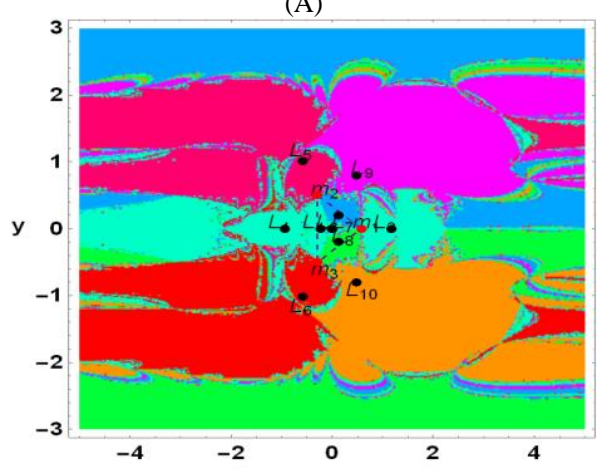

(B)

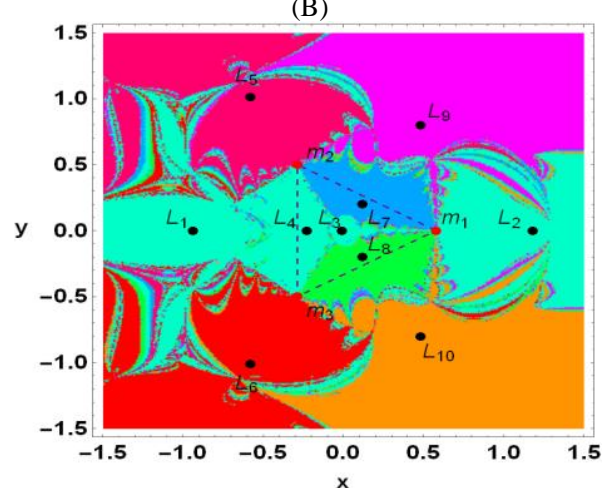

Fig. 6: (A) Basins of Convergence in X-Y-Plane, (B) the Zoomed Part of Figure (A) Near Lagrangian Configuration.

\section{Linear stability}

This section examined the stability of stationary points. For which, we have considered the small perturbation in the equilibrium points $\left(\mathrm{x}_{0}, \mathrm{y}_{0}, \mathrm{z}_{0}\right)$. Let the small displacements be $\left(\mathrm{x}_{0}{ }^{1}, \mathrm{y}_{0}{ }^{1}, \mathrm{z}_{0}{ }^{1}\right)$. Therefore, $\mathrm{x}=\mathrm{x}_{0}+\mathrm{x}_{0}{ }^{1}, \mathrm{y}=\mathrm{y}_{0}+\mathrm{y}_{0}{ }^{1}, \mathrm{z}=\mathrm{z}_{0}+\mathrm{z}_{0}{ }^{1}$.

Now, we can write the variational equations of Equation 3 as:

$$
\begin{aligned}
& \ddot{x}_{0}^{1}-2 \dot{y}_{0}^{1}=x_{0}^{1} \prod_{x x}^{0}+y_{0}^{1} \prod_{x y}^{0}+z_{0}^{1} \Pi_{x z}^{0}, \\
& \ddot{y}_{0}^{1}+2 \dot{x}_{0}^{1}=x_{0}^{1} \Pi_{y x}^{0}+y_{0}^{1} \Pi_{y y}^{0}+z_{0}^{1} \Pi_{y z}^{0}, \\
& \ddot{z}_{0}^{1}=x_{0}^{1} \prod_{z x}^{0}+y_{0}^{1} \prod_{z y}^{0}+z_{0}^{1} \prod_{z z}^{0} .
\end{aligned}
$$

To solve the above variational equation, let $\mathrm{x}_{0}{ }^{1}=\alpha_{1} \mathrm{e}^{\lambda \mathrm{t}}, \mathrm{y}_{0}{ }^{1}=\alpha_{2} \mathrm{e}^{\lambda \mathrm{t}}$ and $\mathrm{z}_{0}{ }^{1}=\alpha_{3} \mathrm{e}^{\lambda \mathrm{t}}$, where $\alpha_{1}, \alpha_{2}, \alpha_{3}$ and $\lambda$ are parameters to be found. Hence

$$
\begin{aligned}
& \alpha_{1}\left(\lambda^{2}-\prod_{x x}^{0}\right)+\alpha_{2}\left(-2 \lambda-\prod_{x y}^{0}\right)+\alpha_{3}\left(-\prod_{x z}^{0}\right)=0, \\
& \alpha_{1}\left(2 \lambda-\prod_{y x}^{0}\right)+\alpha_{2}\left(\lambda^{2}-\prod_{y y}^{0}\right)+\alpha_{3}\left(-\prod_{y z}^{0}\right)=0, \\
& \alpha_{1}\left(-\prod_{z x}^{0}\right)+\alpha_{2}\left(-\prod_{z y}^{0}\right)+\alpha_{3}\left(\lambda^{2}-\prod_{z z}^{0}\right)=0 .
\end{aligned}
$$

The non-trivial solution for $\alpha_{1}, \alpha_{2}$ and $\alpha_{3}$ will be

$$
\begin{aligned}
& \left|\begin{array}{ccc}
\lambda^{2}-\Pi_{x x}^{0} & -2 \lambda-\Pi_{x y}^{0} & -\prod_{x z}^{0} \\
2 \lambda-\prod_{y x}^{0} & \lambda^{2}-\prod_{y y}^{0} & -\prod_{y z}^{0} \\
-\prod_{z x}^{0} & -\prod_{z y}^{0} & \lambda^{2}-\prod_{z z}^{0}
\end{array}\right|=0 \\
& \Rightarrow \quad \lambda^{6}+\beta_{1} \lambda^{4}+\beta_{2} \lambda^{2}+\beta_{3}=0 .
\end{aligned}
$$

Where,

$\beta_{1}=4-\prod_{x x}^{0}-\prod_{y y}^{0}-\prod_{z z}^{0}$,

$\beta_{2}=-\left(\prod_{x y}^{0}\right)^{2}-\left(\prod_{x z}^{0}\right)^{2}-\left(\prod_{y z}^{0}\right)^{2}-4 \prod_{z z}^{0}+\prod_{x x}^{0} \prod_{y y}^{0}+\prod_{x x}^{0} \prod_{z z}^{0}+\prod_{y y}^{0} \prod_{z z}^{0}$,

$\beta_{3}=\left(\prod_{x z}^{0}\right)^{2} \prod_{y y}^{0}+\left(\prod_{y z}^{0}\right)^{2} \prod_{x x}^{0}+\left(\prod_{x y}^{0}\right)^{2} \prod_{z z}^{0}-2 \prod_{x y}^{0} \prod_{x z}^{0} \prod_{y z}^{0}-\prod_{x x}^{0} \prod_{y y}^{0} \prod_{z z}^{0}$.

Numerically, we have evaluated the six roots of the characteristic polynomial from equation (8) for each value of the stationary points and placed in the tables. We observed from the tables that at least one root is either positive real value or positive real part. Therefore all the stationary points are unstable.

Table 1: Corresponding Characteristic Roots of Equilibria in X-Y-Plane

\begin{tabular}{llll}
\hline S. N. & Equilibria $(\mathrm{x}, \mathrm{y})$ & Characteristic Roots & \\
\hline 1 & $-0.94,0$ & $\pm 0.76901241 \pm 0.97034312 \mathrm{i}, \pm 1.14001121 \mathrm{i}$ & Nature \\
2 & $1.18,0$ & $\pm 1.30440257 \mathrm{i}, \pm 1.35333339 \mathrm{i}, \pm 1.23813468$ & Unstable \\
3 & $-0.0125,0$ & $\pm 1.60884467 \pm 0.99381650 \mathrm{i}, \pm 2.28065805 \mathrm{i}$ & Unstable \\
4 & $-0.23,0$ & $\pm 1.94953513 \mathrm{i}, \pm 2.42042221 \mathrm{i}, \pm 2.76751985$ & Unstable \\
5 & $-0.58, \pm 1.012$ & $\pm 1.34672511 \mathrm{i}, \pm 1.39775148 \mathrm{i}, \pm 1.32943265$ & Unstable \\
6 & $0.12, \pm 0.2$ & $\pm 1.96435919 \mathrm{i}, \pm 2.42160170 \mathrm{i}, \pm 2.77900895$ & Unstable \\
\hline
\end{tabular}




$70.48, \pm 0.8 \quad \pm 0.77854509 \pm 0.97090916 \mathrm{i}, \pm 1.15192586 \mathrm{i}$

Table 2: Corresponding Characteristic Roots of Equilibria in Y-Z-Plane

\begin{tabular}{llll}
\hline S. N. & Equilibria $(\mathrm{y}, \mathrm{z})$ & Characteristic Roots & \\
\hline 1 & 0,0 & $\pm 1.61185696 \pm 0.99999999 \mathrm{i}, \pm 2.27950608 \mathrm{i}$ & Nature \\
2 & $\pm 1.063,0$ & $\pm 1.05906266 \mathrm{i}, \pm 1.25993988 \mathrm{i}, \pm 0.84205948$ & Unstable \\
3 & $\pm 0.485,0$ & $\pm 3.66734674 \mathrm{i}, \pm 3.85825462 \mathrm{i}, \pm 5.13186434$ & Unstable \\
\hline
\end{tabular}

Table 3: Corresponding Characteristic Roots of Equilibria in X-Z-Plane

\begin{tabular}{llll}
\hline S. N. & Equilibria $(x, y)$ & Characteristic Roots & Nature \\
\hline 1 & $-0.94,0$ & $\pm 0.76901241 \pm 0.97034312 \mathrm{i}, \pm 1.14001121 \mathrm{i}$ & Unstable \\
2 & 0,0 & $\pm 1.61185696 \pm 0.99999999 \mathrm{i}, \pm 2.27950608 \mathrm{i}$ & Unstable \\
3 & $1.185,0$ & $\pm 1.28940141 \mathrm{i}, \pm 1.33736749 \mathrm{i}, \pm 1.20461941$ & Unstable \\
4 & $-0.24,0$ & $\pm 1.99173439 \mathrm{i}, \pm 2.42257132 \mathrm{i}, \pm 2.79926656$ & Unstable \\
\hline
\end{tabular}

\section{Conclusion}

The behaviour of the test particle (fourth body) has been studied under the influence of the equal heterogeneous oblate spheroid placed at the Lagrangian configuration. We performed the location of stationary points and found ten stationary points from which we observed that, all the stationary points are unstable while in the classical case of the CR4BP, only collinear stationary points were unstable and non-collinear stationary points were stable under some restrictions (Baltagiannis 2011 a). We also illustrate the zero-velocity curves, regions of motion, Poincare surfaces of section and basins of attraction which show the most qualitative dynamical properties of the fourth body.

\section{References}

[1] Abdullah (2014), Periodic orbits around Lagrangian points of the circular restricted four-body problem. Invertis Journal of Science and Technology 7(1), 29-38.

[2] Ansari AA (2016a), Stability of the equilibrium points in the photo-gravitational circular restricted four-body problem with the effect of perturbations and variable mass. Science International (Lahore). 28, 859- 866.

[3] Ansari AA (2016b), Stability of the equilibrium points in the circular restricted four-body problem with oblate primary and variable mass. International Journal of Advanced Astronomy. 4(1), 14-19. https://doi.org/10.14419/ijaa.v4i1.5831.

[4] Ansari AA (2016c), the photo-gravitational circular restricted four-body problem with variable masses. Journal of Engineering and Applied Sciences. 3(2), 30-38.

[5] Ansari AA, Kellil R, Alhussain Z (2017), The effect of perturbations on the circular restricted four-body problem with variable mass. Journal of Mathematics and Computer Science. 17(3), 365-377. https://doi.org/10.22436/jmcs.017.03.03.

[6] Ansari AA, Alhussain Z, Prasad S (2018), The circular restricted three-body problem when both the primaries are heterogeneous spheroid of three layers and infinitesimal body varies it's mass. Journal of Astrophysics and Astronomy. 39, 57. https://doi.org/10.1007/s12036-018-9540-7.

[7] Arribas M, Abad A, Elipe A, Palacios M (2016), Out-of-plane equilibria in the symmetric collinear restricted four-body problem with radiation pressure. Astrophys. Space Sci. 361, 270. https://doi.org/10.1007/s10509-016-2858-1.

[8] Asique MC, et al. (2015a), On the R4BP when third primary is an oblate spheroid. Astrophys. Space Sci. 357, 82(1), https://doi.org/10.1007/s10509-015-2235-5.

[9] Asique MC, et al. (2015b), on the photo gravitational R4BP when the third primary is an oblate/prolate spheroid. Astrophys. Space Sci. 360, 13(1), https://doi.org/10.1007/s10509-015-2522-1.

[10] Asique MC, et al. (2016), on the photo-gravitational R4BP when the third primary is a tri-axial rigid body. Astrophys. Space Sci. 361, 379, https://doi.org/10.1007/s10509-016-2959-x

[11] Asique MC, et al. (2017), On the R4BP when Third Primary is an Ellipsoid. Journal of Astronaut. Sci. 64, 231-250, https://doi.org/10.1007/s40295-016-0104-2

[12] Baltagiannis A, Papadakis KE (2011a), Equilibrium points and their stability in the restricted four body problem. International Journal of Bifurcation and Chaos. 21(8), 2179-2193, https://doi.org/10.1142/S0218127411029707.

[13] Baltagiannis AN, Papadakis KE (2011b), Families of periodic orbits in the restricted four-body problem. Astrophys. Space Sci. 336, 357-367. https://doi.org/10.1007/s10509-011-0778-7.

[14] Ceccaroni M, Biggs J (2012), Low-thrust propulsion in a coplanar circular restricted four body problem. Celest. Mech. Dyn. Astron. 112,191-219. https://doi.org/10.1007/s10569-011-9391-X

[15] Esteban EP, Vazquez S (2001), Rotating stratified heterogeneous oblate spheriod in Newtonian Physics, Celestial Mechanics and Dynamical Astronomy. 81, 299 - 312. https://doi.org/10.1023/A:1013292529030.

[16] Idrisi J and Shalini K (2016), Non-collinear libration points in CR3BP when less massive primary is an heterogeneous oblate body with N-layers International Journal of Advanced Astronomy. 4(1), 39-42. https://doi.org/10.14419/ijaa.v4i1.5928.

[17] Kalvouridis TJ, Arribas M, Elipe A (2006a), Dynamical properties of the restricted four-body problem with radiation pressure. Mechanics research communications. 33, 811-817. https://doi.org/10.1016/i.mechrescom.2006.01.008

[18] Kalvouridis T, Arribas M, Elipe A (2006b), the photo-gravitational restricted four-body problem: an exploration of its dynamical properties. AIP Conf. Proc., 848, 637. https://doi.org/10.1063/1.2348041.

[19] Kumari R, Kushvah BS (2013), Equilibrium points and zero velocity surfaces in the restricted four-body problem with solar wind drag. Astrophys. Space Sci. 344, 347-359. https://doi.org/10.1007/s10509-012-1340-y.

[20] Kumari R, Kushvah BS (2014), Stability regions of equilibrium points in the restricted four-body problem with oblateness effects. Astrophys. Space Sci. 349, 693-704. https://doi.org/10.1007/s10509-013-1689-6.

[21] Papadakis KE (2007), Asymptotic orbits in the restricted four-body problem. Planet. Space Sci., 55, 1368. https://doi.org/10.1016/j.pss.2007.02.005.

[22] Papadakis KE (2016a), Families of three-dimensional periodic solutions in the circular restricted four-body problem. Astrophys. Space Sci. 361, 129. https://doi.org/10.1007/s10509-016-2713-4.

[23] Papadakis KE (2016b), Families of asymmetric periodic solutions in the restricted four-body problem. Astrophys. Space Sci. 361, 377. https://doi.org/10.1007/s10509-016-2965-Z.

[24] Papadouris JP, Papadakis KE (2013), Equilibrium points in the photo-gravitational restricted four-body problem. Astrophys. Space Sci. 344, 21-38. https://doi.org/10.1007/s10509-012-1319-8.

[25] Papadouris JP, Papadakis KE (2014), Periodic solutions in the photo-gravitational restricted four-body problem. MNRAS. 442, 1628-1639. https://doi.org/10.1093/mnras/stu981. 
[26] Shalini K, et al. (2017), the non-linear stability of $\mathrm{L}_{4}$ in the R3BP when the smaller primary is a heterogeneous spheroid. J. Astronaut. Sci., 64 (1), 18-49. https://doi.org/10.1007/s40295-016-0093-1.

[27] Singh J, Vincent AE (2015a), Out-of-plane equilibrium points in the photo-gravitational restricted four-body problem. Astrophys. Space Sci. 359, 38. https://doi.org/10.1007/s10509-015-2487-0.

[28] Singh J, Vincent AE (2015b), Effect of perturbations in the Coriolis and centrifugal forces on the stability of equilibrium points in the restricted four-body problem. Few-Body Syst. 56, 713-723. https://doi.org/10.1007/s00601-015-1019-3.

[29] Singh J, Vincent AE (2016a), Equilibrium points in the restricted four-body problem with radiation pressure. Few-Body Syst. 57, 83-91. https://doi.org/10.1007/s00601-015-1030-8.

[30] Singh J, Vincent AE (2016b), Out-of-plane equilibrium points in the photo-gravitational restricted four-body problem with oblateness. British Journal of Mathematics and Computer Science. 19(5), 1-15. https://doi.org/10.9734/BJMCS/2016/29698

[31] Suraj MS, Hassan MR, Chand MA (2014), the photo gravitational R3BP when the primaries are heterogeneous spheroid with three layers. J. Astronaut. Sci. 61 (2), 133. https://doi.org/10.1007/s40295-014-0026-9.

[32] Suraj MS, Aggarwal R, Arora M (2017), On the restricted four-body problem with the effect of small perturbations in the Coriolis and centrifugal forces. Astrophys. Space Sci. 362, 159. https://doi.org/10.1007/s10509-017-3123-y.

[33] Suraj MS, et al. (2017), Fractal basins of attraction in the restricted four-body problem when the primaries are tri-axial rigid bodies. Astrophys. Space Sci. 362: 211. https://doi.org/10.1007/s10509-017-3188-7.

[34] Zotos EE (2017), revealing the basins of convergence in the planar equilateral restricted four-body problem. Astrophys. Space Sci. 362(2). https://doi.org/10.1007/s10509-017-3172-2. 